\title{
Direct Numerical Simulation of Receptivity to Roughness in a Swept-Wing Boundary Layer at High Reynolds Numbers
}

\author{
Gary Nicholson*, Chao Zhang ${ }^{\dagger}$, Lian Duan ${ }^{\ddagger}$ \\ Missouri University of Science and Technology, Rolla, MO 65409 \\ Mujeeb Malik ${ }^{\S}$, Fei Li ${ }^{\uparrow}$ \\ NASA Langley Research Center, Hampton, VA 23681 \\ Ali Uzunl \\ National Institute of Aerospace, Hampton, VA 23666
}

\begin{abstract}
Direct numerical simulations (DNS) are performed to examine the receptivity to roughness in a spatially developing three-dimensional boundary layer over an infinite-swept natural-laminar-flow wing at a freestream Mach number of 0.75 and a chord Reynolds number of approximately 25 million based on the long, swept chord. Stationary crossflow disturbances are excited by applying either critically spaced discrete cylinders of micron size or naturally occurring distributed roughness in the leading-edge region. The DNS data show that the spanwise spectral content of the excited crossflow disturbances is highly dependent upon the shape of roughness elements, and the initial growth of the crossflow structures is a nonlinear function of the element height. The linear growth rate of the excited crossflow disturbances predicted by DNS shows good agreement with linear parabolized stability equations. The receptivity study lays the foundation for investigating the stabilization of the naturally most unstable steady crossflow mode by using spanwise periodic DREs.
\end{abstract}

\section{Nomenclature}

speed of sound, $\mathrm{m} / \mathrm{s}$ unswept chord measured in direction perpendicular to leading edge, $\mathrm{m}$ long, swept chord measured in direction parallel to the incoming freestream velocity, $c_{s}=c / \cos (\Lambda)$, m diameter of cylindrical roughness elements, $\mathrm{m}$ height of cylindrical roughness elements, $m$ pressure coefficient, $C_{p}=\left(p-p_{\infty}\right) /\left(0.5 \rho_{\infty} Q_{\infty}^{2}\right)$, dimensionless freestream Mach number, $M_{\infty}=Q_{\infty} / a_{\infty}$, dimensionless Reynolds number based on unswept chord $c, R e_{c} \equiv \rho_{\infty} Q_{\infty} c / \mu_{\infty}$, dimensionless Reynolds number based on swept chord $c_{s}, R e_{c_{s}} \equiv \rho_{\infty} Q_{\infty} c_{s} / \mu_{\infty}$, dimensionless free flight velocity, $\mathrm{m} / \mathrm{s}$ temperature, $\mathrm{K}$ chordwise velocity, $\mathrm{m} / \mathrm{s}$ spanwise velocity, $\mathrm{m} / \mathrm{s}$

\footnotetext{
*Graduate Research Assistant, Student member, AIAA

$\dagger$ Graduate Research Assistant, Student member, AIAA

$\ddagger$ Assistant Professor. Senior member, AIAA

$\S$ Senior Aerodynamicist, Computational AeroSciences Branch, M.S. 128. Fellow, AIAA

ฯ Aerospace Technologist, Computational AeroSciences Branch, M.S. 128

$\|$ Senior Research Scientist. Senior member, AIAA
} 
$w$ normal-to-the-chord velocity, $\mathrm{m} / \mathrm{s}$

$x \quad$ Cartesian coordinate in the chordwise direction perpendicular to the leading edge of the swept wing

$y \quad$ Cartesian coordinate in the spanwise direction parallel to the leading edge of the swept wing

$z \quad$ Cartesian coordinate normal to the chord

$i \quad$ curvilinear coordinate along the vortex axis in the nonorthogonal system

$j \quad$ curvilinear coordinate parallel to the leading edge in the nonorthogonal system

$k \quad$ curvilinear coordinate defining the wall-normal direction in the nonorthogonal system

$L_{r} \quad$ spacing of the spanwise array of roughness elements, $\mathrm{m}$

$L_{y} \quad$ spanwise domain size of DNS, m

$A_{s} \quad$ modal amplitude of the chordwise velocity perturbation $A_{s}=\max _{k}\left|\hat{u}_{s}\right|, \mathrm{m} / \mathrm{s}$

$N \quad$ logarithmic-amplification ratio, $N=\log \left(A_{s}(i) / A_{s}\left(i_{0}\right)\right)$, dimensionless

$\Lambda \quad$ wing sweep angle, degree

$\beta_{0} \quad$ fundamental disturbance spanwise wave number, $\mathrm{m}^{-1}$

Subscripts

$r \quad$ quantities related to roughness

rms root mean square

w wall variables

$\infty \quad$ freestream variables

Superscripts

$+\quad$ inner wall units

$\overline{(\cdot)}$ unperturbed baseflow variables

$(\cdot)^{\prime}$ perturbation from the baseflow due to roughness

$(\cdot)^{*}$ complex conjugate

$(\hat{.}) \quad$ spanwise Fourier transformed variables

\section{Introduction}

Skin-friction drag accounts for approximately one-half of the total drag for business jets and long-haul transport aircraft. Transition delay via laminar flow technology is an important component of drag reduction technologies. Although system studies have shown that 9-10\% fuel savings can be achieved by delaying boundary-layer transition over major aerodynamic surfaces, the projected benefits can be significantly offset by uncertainties in transition prediction. To enable usable and robust designs for Natural Laminar Flow (NLF) and Hybrid Laminar Flow Control (HLFC), linking transition prediction to high-fidelity aircraft design tools is critical. One important technical gap that prevents accurate transition prediction in sweptwing boundary layers is the transition due to crossflow instability, which is extremely sensitive to surface roughness, especially near the wing leading edge. ${ }^{1,2}$

In three-dimensional swept-wing boundary layers, crossflow instability often manifests itself in the form of stationary corotating streamwise vortices that originate at minute roughness sites. Amplitude of the induced stationary disturbances is directly related to the leading-edge surface finish. It has been found that the growth of these crossflow disturbances can be delayed by placing discrete roughness elements (DREs) of subcritical wavelengths near the wing leading edge. ${ }^{3,4}$ The effectiveness of the DRE concept has been demonstrated in a bulk of the existing experimental and computational studies, and these studies were carried out for low-Mach-number $\left(M_{\infty}<0.3\right)$ configurations with modest wing-chord Reynolds numbers of up to approximately $R e_{c_{s}}=8 \times 10^{6}, 1,2,5-10$ and with pressure distributions that may not be optimal for wing designs for subsonic transport aircraft flying at Mach numbers between 0.75 and 0.90 .

To further assess the potential capability of the DRE concept to control swept-wing transition at transonic Mach numbers and substantially higher chord Reynolds numbers than previous applications, a high-Reynolds-number flight experiment, referred to as the Subsonic Aircraft Roughness Glove Experiment (SARGE), was recently initiated to design high-Reynolds-number NLF wing configurations with a maximum possible chord Reynolds number approaching $R e_{c_{s}}=30 \times 10^{6} .{ }^{11}$ For such configurations, Malik et al. ${ }^{12}$ and Li et al. ${ }^{13}$ conducted a computational assessment of the DRE concept using nonlinear parabolized stability equations (PSE) and secondary-instability analysis, with the particular conditions used for the assessment consisting of a freestream Mach number of 0.75 and chord Reynolds numbers of $17 \times 10^{6}, 24 \times 10^{6}$, and $30 \times 10^{6}$. The computations demonstrated that DREs can suppress dominant boundary-layer disturbances 
at the chosen Reynolds numbers. However, in their calculations the receptivity phase was not incorporated. Instead, a linear eigenmode was used to initialize the calculation while the impact of the actual surface roughness to initiate natural crossflow disturbances, as well as the control mode, was not simulated. Given that it is not known how far downstream of the DREs a crossflow eigenmode shape develops and what the relation of its amplitude to the height and shape of the roughness is, a further study of receptivity to roughness (i.e., the relation between the size and/or distribution of roughness and induced stationary disturbance in the boundary-layer) is needed for given DRE height, shape, and location in order to draw definitive conclusions on whether DREs can delay crossflow-induced transition.

Direct numerical simulation (DNS) is a valuable tool that can be combined with stability analysis to estimate the range of amplitudes of the stationary-crossflow instabilities excited by surface roughness. DNS can be used to elucidate features of roughness-induced flow fields ${ }^{8,14}$ and validate reduced-order receptivity models such as those based on PSE ${ }^{15}$ and linearized Navier-Stokes equations. ${ }^{16,17}$ For instance, Tempelmann et al. ${ }^{15}$ studied receptivity to DREs using a combination of DNS and PSE with the flow conditions corresponding to wind-tunnel experiments by Saric and coworkers at Arizona State University (ASU). ${ }^{18}$ In their study, DNS solutions were used to provide insight into different roughness parameter effects and to validate a receptivity model based on direct and adjoint PSEs. Rizzetta et al. ${ }^{8}$ studied the excitation of crossflow instabilities with critically spaced DREs placed near a swept-wing leading edge, with flow configuration corresponding to the low-speed swept-wing flight test (SWIFT) by the Texas A\&M University. ${ }^{1,2}$ They found that the initial growth of the velocity amplitude displayed a nonlinear dependence on elemental height, indicating the necessity for performing a DNS for each roughness configuration in order to obtain meaningful perturbation quantities that may then be used to carry out stability calculations. The DNS were also fed into the downstream region as initial conditions for nonlinear PSE (NPSE) computations and the combined NPSE and DNS approach were found to produce nearly identical initial growth rates as the full DNS. As far as transition control with DREs is concerned, Wasserman and Kloker ${ }^{7}$ conducted a DNS study of control of crossflow vortices in a three-dimensional (3-D) low-speed flat-plate boundary layer. Hosseini et al. ${ }^{10}$ further conducted DNS to study the stabilization of a swept-wing boundary layer by DREs with flow conditions representative of the ASU experiments. Both studies concluded that DREs stabilizes the primary crossflow modes and attenuates the growth of secondary instabilities. So far, none of the previous DNS of receptivity to DREs were carried out with Mach number, Reynolds number, and pressure distributions that are relevant to subsonic transport aircraft.

To complement the stability analysis by Malik et al. ${ }^{12}$ and Li et al. ${ }^{13}$ as well as to provide computational assessment of the DRE technology for potential application to transport aircraft, the current paper studies the receptivity to roughness using DNS of compressible Navier-Stokes equations for a spatially developing transonic 3-D boundary layer over a realistic NLF wing configuration at Reynolds numbers relevant to transport aircraft. DNS results are analyzed to determine the initial amplitude and mode shapes of induced stationary crossflow modes as well as to shed light on the relation of the modal amplitudes to the size and distribution of roughness.

The paper is structured as follows. The flow conditions and numerical methods are outlined in Section II. Section III presents DNS results of the baseflow and the growth of roughness-induced disturbances. Section IV gives a summary of the current study.

\section{Flow Conditions and Numerical Methodology}

The work in this paper considers the boundary layer over a swept NLF wing (G-IIB, TAMU-0706 wing glove) designed by Tufts et al. ${ }^{19}$ at Texas A \& M University. The design of TAMU-0706 wing glove has improved upon that of Belisle et al. ${ }^{11}$ with a truly uniform flow in the spanwise direction and substantially improved stability characteristics. The target design conditions consist of $M_{\infty}=0.75$ at an altitude of $H=40 \mathrm{kft}$, an angle of attack (AoA) of 3.7 degrees, a chord Reynolds number, $R e_{c_{s}}$, of 24.77 million, and a leading-edge sweep angle, $\Lambda$, of $30^{\circ}$. The free flight conditions for the work described in this paper are summarized in Table 1. In this paper, we focus on an infinite-swept TAMU-0706 wing at an AoA of 1.9375 degrees. For the selected angle of attack, the surface pressure coefficient $C_{p}$ near the leading edge of the infinite-swept wing matches as closely as possible that of the three-dimensional (3-D) finite-swept counterpart at the design AoA of 3.7 degrees (See Figure 5a in Section III).

For the selected configuration, two types of DNS are carried out. A DNS without roughness, referred to as DNS-I, is first computed to simulate the steady baseflow that serves to provide boundary conditions 
Table 1. Free flight conditions for the DNS.

$\begin{array}{ccccccc}M_{\infty} & Q_{\infty}(\mathrm{m} / \mathrm{s}) & \rho_{\infty}\left(\mathrm{kg} / \mathrm{m}^{3}\right) & T_{\infty}(\mathrm{K}) & c(\mathrm{~m}) & \operatorname{Re}_{c}\left(\times 10^{6}\right) & \operatorname{Re}_{c_{s}}\left(\times 10^{6}\right) \\ 0.75 & 221.28 & 0.302 & 216.65 & 3.83 & 21.45 & 24.77\end{array}$

for a second DNS that includes roughness, referred to as DNS-II. To select a suitable domain for DNS-I, a precursor Reynolds-averaged Navier-Stokes (RANS) simulation of the flow for the entire wing is carried out and the flow field is explored. In the RANS, the Spalart-Allmaras turbulence model is used; and the flow is assumed to be homogeneous in the spanwise direction. The flow is set to be laminar over the first $61 \%$ of the wing on both the upper and lower surfaces and the turbulence model is only switched on outside of the laminar region. In DNS-II, surface roughness is incorporated to excite crossflow disturbances. The roughness elements implemented in the DNS include a spanwise periodic row of discrete circular cylinders of various height and diameters that is formed by displacing the corresponding mesh points at the wall (Section B) and naturally occurring distributed roughness that is modeled by an appropriate roughness model combined with inhomogeneous boundary conditions at the undisturbed wall (Section C).

The wing and the adopted coordinate systems are shown in Figure 1. A nonorthogonal coordinate system is used for the current DNS. In this coordinate system, the body-fitted curvilinear computational coordinate $i$ is approximately aligned with the crossflow vortex axis rather than along the chordwise direction ( $x$ direction). By doing so, the number of grid points, which is required in the streamwise direction due to the relatively slow evolution of crossflow modes along this direction, can be substantially reduced. The spanwise computational coordinate $j$ is along the $y$ direction, which is parallel to the leading edge of the wing and at an acute angle to the $i$-coordinate. Similar nonorthogonal systems have been chosen in spatially developing secondary instability analyses ${ }^{20}$ and $\mathrm{DNS}^{21,22}$ for crossflow-dominated swept-wing boundary layers. Both DNS-I and DNS-II simulate the physical boundary layer over the TAMU wing extending approximately from $x / c=0.006$ on the wing lower surface or windward side to $x / c=0.7$ on the wing upper surface or leeward side. Only the flow field on the leeward side is of interest, while part of the windward side is retained to account for the asymmetry of the configuration. Negligible differences have been observed when the domain is further enlarged on the wing lower surface. The domain size in the spanwise direction is set to be equal to the spacing $L_{r}$ of the spanwise array of roughness elements. Sponge regions are inserted in both types of DNS in order to minimize acoustic reflections at the lower and upper outlets and at the freestream boundary (Figure 2). Within these sponge regions, the flow is forced towards the RANS solution for the entire TAMU wing. On the wall, no-slip conditions are applied for the three velocity components and the temperature is extracted from the RANS solution in which an adiabatic condition is used; and the extracted wall temperature is prescribed as Dirichlet conditions in the DNS.

To simulate the boundary layer flow over the domain, the compressible Navier-Stokes equations are solved in generalized curvilinear coordinates. The working fluid is assumed to be an ideal gas with a linear (i.e., Newtonian) stress-strain relation. The Fourier law is used to compute the heat flux terms. A 7th-order weighted essentially nonoscillatory (WENO) scheme $^{23}$ is used to compute the convective flux terms. Given the subsonic nature of the flow without shock waves, the optimal 9-point WENO stencil is used in the simulations with WENO adaptation turned off to reduce numerical dissipation. For the viscous flux terms, a 4th-order central difference scheme is used. The 3rd-order low-storage Runge-Kutta scheme by Williamson ${ }^{24}$ is used for time integration.

The total number of grid points is 4949,320 , and 295 in the streamwise $(i)$, spanwise $(j)$, and wallnormal $(k)$ directions, respectively. The grid is clustered near the wall and close to the roughness element (Figure 3). The computational grid resolution is comparable to those reported in the literature in the context of previous simulations of turbulent wall-bounded flows using comparable numerical algorithms. ${ }^{22}$ Moreover, a grid convergence study shows that negligible differences are observed for the reported quantities by further refining the meshes in each direction, as illustrated in Figure 4. 


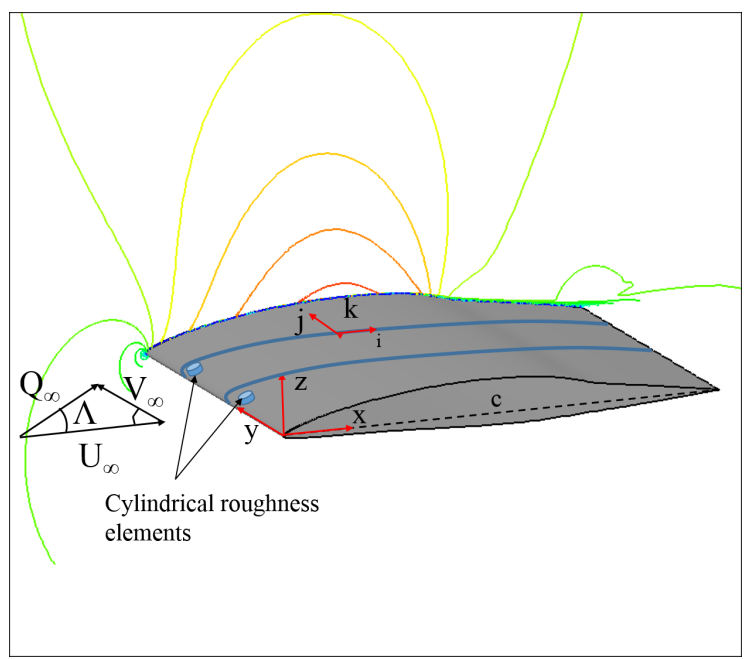

Figure 1. Swept TAMU-0706 wing, with sweep angle $\Lambda=30^{\circ}$ and the total incoming velocity $Q_{\infty}$. The wing is at an angle-of-attack of $1.9375^{\circ}$. Here, $(i, j, k)$ and $(x, y, z)$ represent locally fitting curvilinear and Cartesian coordinate systems, respectively. The colored contours denote the chordwise velocity $(\bar{u})$ from the RANS. The blue lines represent the crossflow vortex axis.

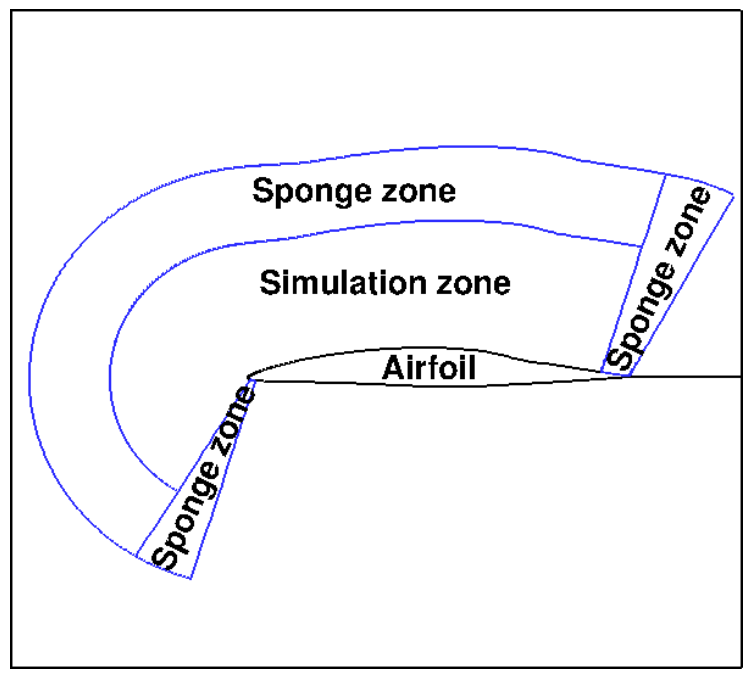

Figure 2. Sketch of the computational domain and boundary conditions for the DNS simulation. 


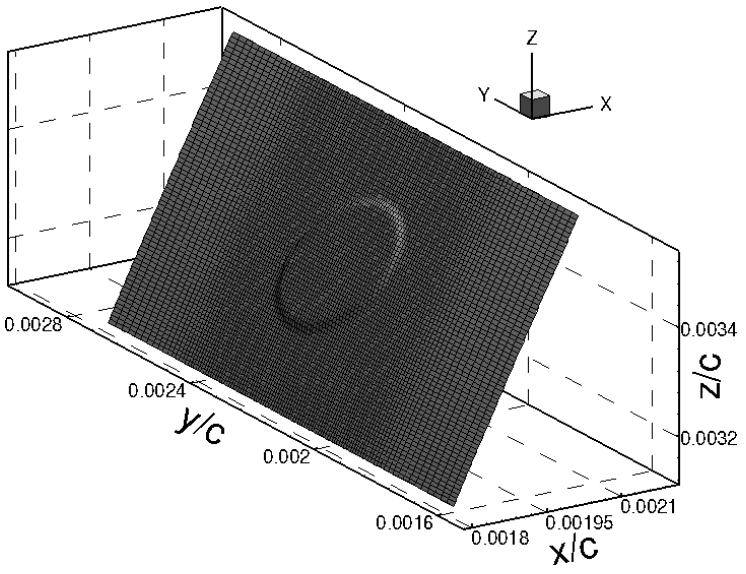

(a)

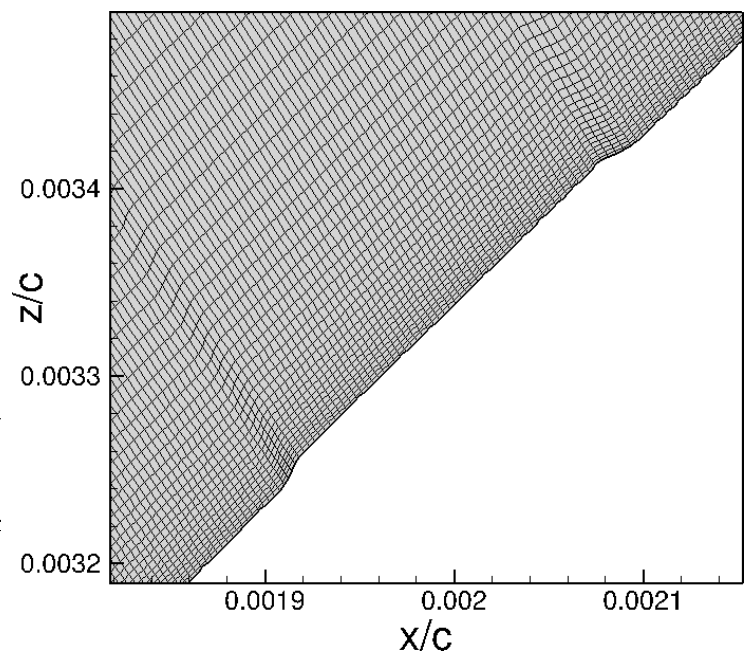

(b)

Figure 3. (a) Meshed cylindrical roughness element generated by displacing the corresponding mesh points at the wall; (b) A close-up of the mesh in a x-z plane $\left(y=0.5 L_{r}\right)$ near the roughness element.

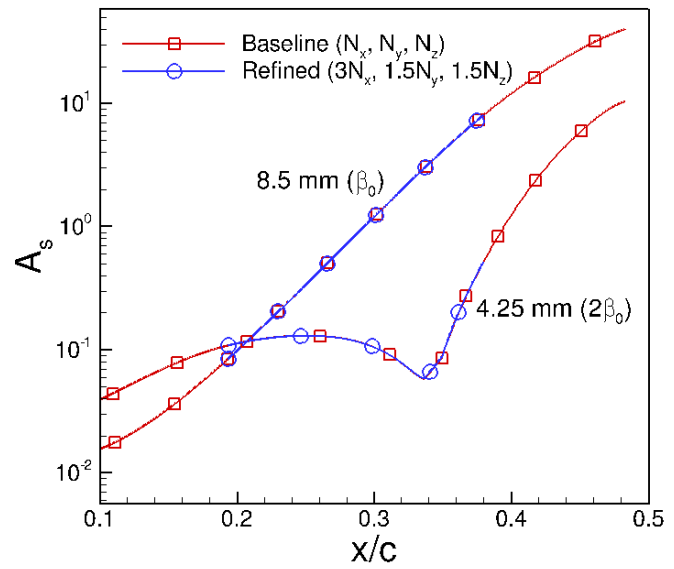

Figure 4. Grid convergence study for chordwise evolution of disturbance amplitudes $A_{s}$ of the fundamental mode $\beta_{0}=2 \pi / L_{r}$ and the first superharmonic $2 \beta_{0}$ for Case Cyl_D1_H10_L8p5. $A_{s}$ is extracted from the DNS data by means of Fourier transform in the spanwise direction. 


\section{Results}

In this Section, the validity of the DNS-computed baseflow of the infinite-swept NLF wing is first verified; next, DNS results of receptivity to localized and natural surface roughness on the wing are introduced; finally, the stabilization of crossflow instabilities with subcritical DREs is simulated by the DNS.

\section{A. Baseflow Results}

To check the validity of the baseflow computed by the DNS, Figures $5 \mathrm{a}$ and $5 \mathrm{~b}$ show comparisons in surface pressure coefficient and the density contours, respectively, between the full-wing RANS and DNS-I that consists of a partial wing. Excellent comparison between the RANS and the DNS is achieved for both quantities. In particular, Figure 5a shows that the RANS and DNS results for the infinite-swept wing closely match that of the 3D finite-swept wing with an AoA of 3.7 degrees for $x / c<0.45$. Small differences in $C_{p}$ exist over the leading edge between the 3D and the infinite-swept wings. Such differences might be due to the slight geometric smoothing applied to the leading-edge region or due to the infinite-swept-wing assumption.

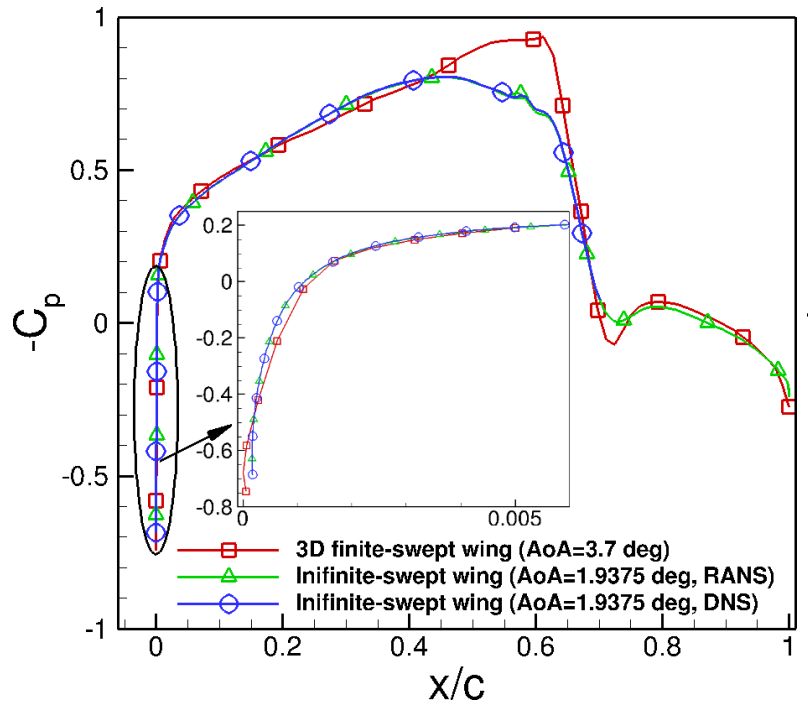

(a)

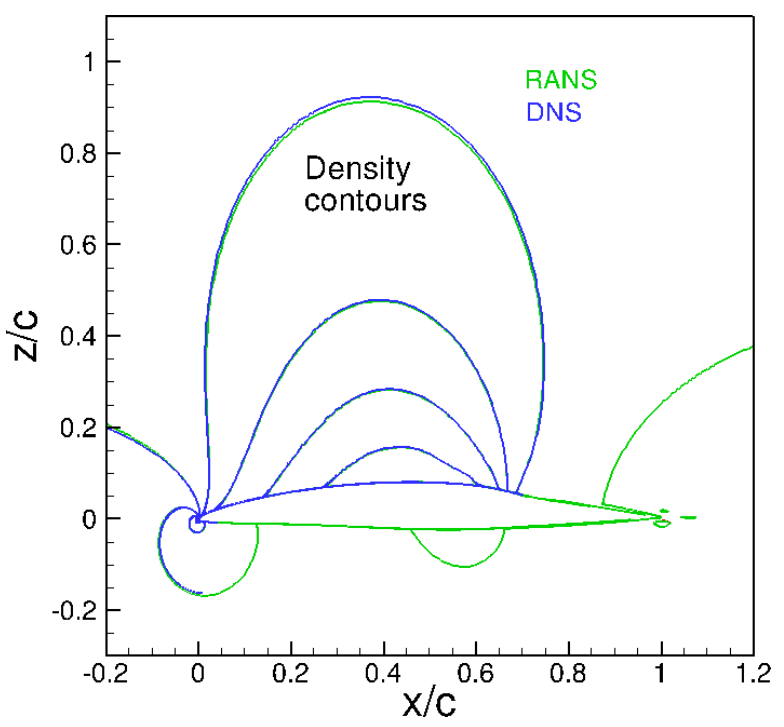

(b)

Figure 5. (a) Surface pressure coefficient $C_{p}$ obtained from the DNS and RANS for the infiniteswept wing (without roughness) in comparison with the results in Tufts et al. ${ }^{19}$ The inset provides a zoomed-in view of all $C_{p}$ curves over the leading edge. (b) Density contours of the baseflow obtained from the RANS calculation of the entire wing and the current DNS.

\section{B. Receptivity to Discrete Surface Roughness}

Next, DNS results of receptivity to a spanwise array of micron-sized circular cylinders are presented. In each DNS, stationary crossflow modes are excited by inserting a single roughness element in the form of a shallow cylindrical disk near the leading edge, and spanwise periodic boundary conditions are prescribed to mimic a row of spanwise periodic roughness. The use of a single roughness in the DNS domain with spanwise periodic boundary conditions has been shown to be adequate for modeling a spanwise cyclic roughness array. ${ }^{15}$ The roughness is inserted into the computational grid by deforming the baseline grid to accommodate the change in surface geometry as shown in Figure 3. The edges of cylindrical roughness elements are slightly rounded to avoid singularities in computations of grid metrics. Table 2 lists roughness parameters and provides an overview of the cases. The spanwise roughness spacing $L_{r}$ is chosen to be $8.5 \mathrm{~mm}$, corresponding to the wavelength of the naturally most unstable stationary crossflow mode. The roughness elements are placed near the leading edge at $0.2 \%$ of the chord $(x / c=0.002)$. Different roughness heights and diameters are used to provide insight into the sensitivity of receptivity to roughness parameters. 
Table 2. Overview of DNS cases for studying receptivity to localized surface roughness together with the respective roughness parameters.

\begin{tabular}{ccccc}
\hline Case & Location & Diameter & Height & Spacing \\
& $x_{r} / c$ & $d_{r}(\mathrm{~mm})$ & $h_{r}(\mu \mathrm{m})$ & $L_{r}(\mathrm{~mm})$ \\
Cyl_D1_H10_L8p5 & 0.002 & 1 & 10 & 8.5 \\
Cyl_D2_H10_L8p5 & 0.002 & 2 & 10 & 8.5 \\
Cyl_D1_H20_L8p5 & 0.002 & 1 & 20 & 8.5 \\
\hline
\end{tabular}

Figure 6 depicts various planes of the total chordwise velocity $\left(\left(\bar{u}+u^{\prime}\right) / Q_{\infty}\right)$ extracted from the flow field downstream of the cylindrical roughness element $\left(d_{r}=1 \mathrm{~mm}\right.$ and $\left.h_{r}=10 \mu \mathrm{m}\right)$. Spanwise variations of the flow field become apparent for $x / c>0.42$, suggesting that the excited disturbance has reached an amplitude of the order of the baseflow at this location. The overturning flow structures for $x / c>0.42$ are characteristic of crossflow disturbances and conform to those observed by previous studies. ${ }^{5,6,18}$

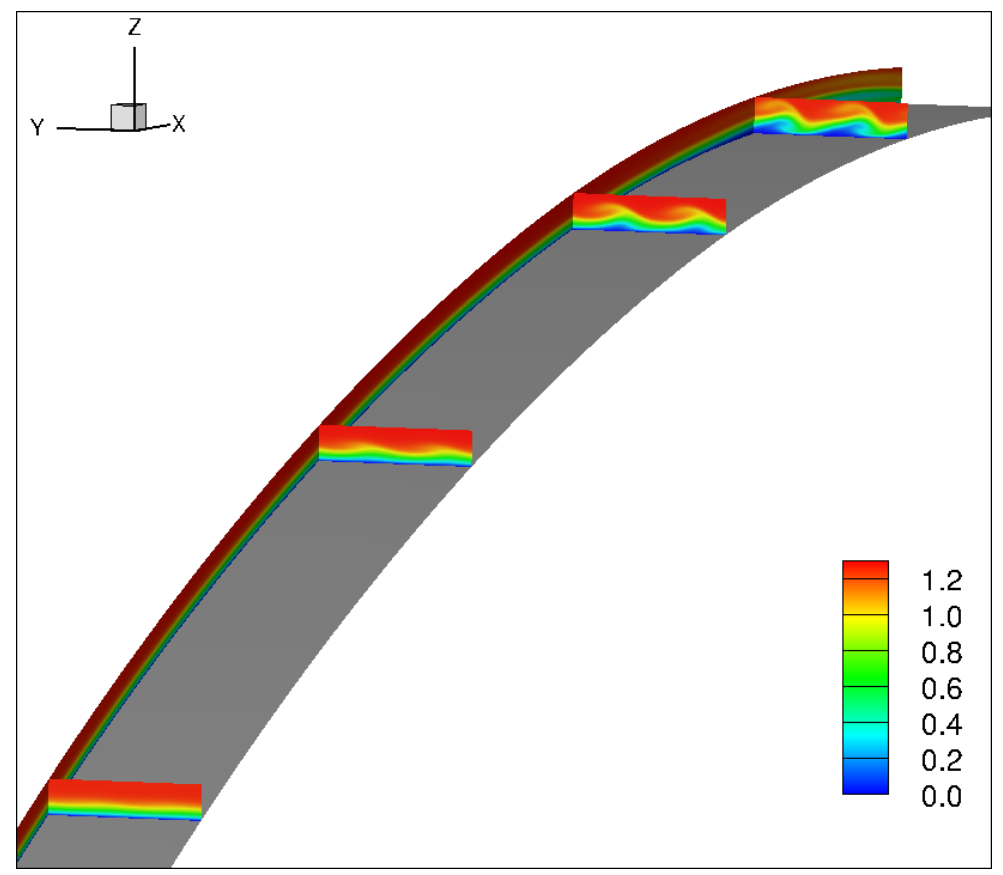

Figure 6. Planes of the total velocity field $\left(\bar{u}+u^{\prime}\right) / Q_{\infty}$ as predicted by DNS for roughness element with $d_{r}=1 \mathrm{~mm}$ and $h_{r}=10 \mu \mathrm{m}$ (Case Cyl_D1_H10_L8p5). The four slices of pseudocolors are located at $x / c=0.35,0.42,0.48,0.53$. The flow is duplicated periodically in the spanwise direction.

Figure 7 plots the r.m.s. of the chordwise velocity disturbances $u_{r m s}^{\prime} / Q_{\infty}$ as a function of $x / c$ for the three roughness cases. As expected, the disturbance magnitude increases as the roughness size becomes larger. Similar increases in $u_{r m s}^{\prime} / Q_{\infty}$ are shown by either doubling the roughness height or by doubling the diameter. For the baseline roughness case with $d_{r}=1 \mathrm{~mm}$ and $h_{r}=10 \mu \mathrm{m}, u_{r m s}^{\prime} / Q_{\infty}$ becomes approximately $7 \%$ at $x / c=0.42$, which suggests a nonlinear interaction and is consistent with the apparent appearance of overturning-wave structures shown in Figure 6.

To characterize the initial spanwise content of the roughness-induced perturbations, Figure 8 plots the initial spanwise wavenumber spectra of the maximum chordwise velocity perturbation at multiple streamwise locations $x / c$. The modal amplitude $A_{s}$ is extracted from the DNS data by means of Fourier transform in the spanwise direction, with the first (or fundamental) mode corresponding to the fundamental interelement spacing $L_{r}=8.5 \mathrm{~mm}$. Immediately downstream of the roughness at $x / c=0.004$, there exists a wide range of spanwise modes, and the initial spectral content is highly sensitive to the diameter and height of the roughness elements. Most of the small-wavelength disturbances decay over relatively short disturbances and 


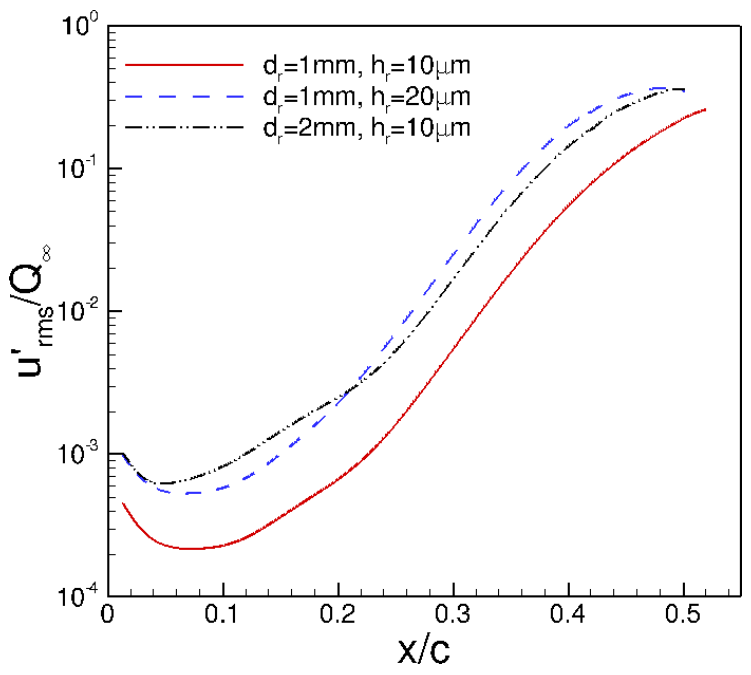

Figure 7. Chordwise evolution of crossflow disturbances for cylindrical roughness with different parameters (Cases Cyl_D1_H10_L8p5, Cyl_D2_H10_L8p5, Cyl_D1_H20_L8p5) as predicted by DNS.

nonnegligible contributions to the spectral content is observed only for the first five modes after $x / c=0.05$. Shown in Figure 9 are the normalized spanwise wavenumber spectra, where the amplitude of each mode has been normalized by the corresponding amplitude for the baseline case with $d_{r}=1 \mathrm{~mm}, h_{r}=10$ $\mu \mathrm{m}$ (Case Cyl_D1_H10_L8p5). Thus, all the normalized amplitudes are identically equal to 1.0 for Case Cyl_D1_H10_L8p5, and the normalized values would be 2.0 for Cases Cyl_D1_H20_L8p5 and Cyl_D2_H10_L8p5 if the amplitudes varied linearly with element height and diameter. Figure 9 shows that the initial amplitude of the crossflow disturbances is a nonlinear function of the elemental height and diameter, although a roughly linear dependence has been found for the r.m.s of chordwise velocity perturbation in Figure 7 . The nonlinear dependence of modal amplitude on roughness parameters is consistent with the finding of Rizzetta et al. in their DNS study of receptivity to DREs over the SWIFT configuration. ${ }^{8}$ Figure 10 further shows the downstream evolution of modal amplitudes, $A_{s}$, of the fundamental mode $\beta_{0}=2 \pi / L_{r}$ and the first four superharmonics for all cylindrical roughness elements. The fundamental 8.5-mm mode $\left(\beta_{0}\right)$ grows continuously through the domain, as expected, and becomes dominant after $x / c=0.25$. The other modes also grow initially, but modes decay further downstream before nonlinear interaction kicks in at $x / c \approx 0.35$. A similar trend has also been reported by Tempelmann et al. ${ }^{15}$ in their study of receptivity to DREs for the SWIFT configuration. The significant initial growth of the $4.25-\mathrm{mm}$ mode $\left(2 \beta_{0}\right)$ and the $2.833-$ mm mode $\left(3 \beta_{0}\right)$ may suggest that either mode could be used as a "control mode" for controlling the most unstable 8.5-mm mode.

Figure 11 shows a comparison in N-factors of the fundamental mode $\beta_{0}$ and the first superharmonic $2 \beta_{0}$ between the DNS and the linear PSE. Good agreement is achieved between the results of DNS and PSE for $0.1<x / c<0.28$. The discrepancies between DNS and linear PSE results for $x / c>0.28$ may be due to nonlinear interaction or additional nonmodal effects in the DNS that are not included in the linear PSE calculation.

\section{Receptivity to Distributed Natural Surface Roughness}

Further, the emergence of unstable crossflow modes due to the presence of natural surface roughness in the leading edge region is computed by DNS. We simulate the effect of natural surface roughness following a procedure proposed by Hosseini et al., ${ }^{10}$ in which roughness is modeled by covering the leading-edge region of the infinite-swept wing with a spanwise periodic roughness strip of the form

$h_{r}(x, y)=\epsilon_{r} H_{\beta}(x) \sum_{n=1}^{M N} \sin \left(n \beta_{0} y+\varphi_{n}\right), \quad$ with $\quad \beta_{0}=2 \pi / L_{y}, \quad H_{\beta}(x)=\left[S\left(\frac{x-x_{s}}{L_{1}}\right)-S\left(\frac{x-x_{e}}{L_{2}}+1\right)\right]$. 


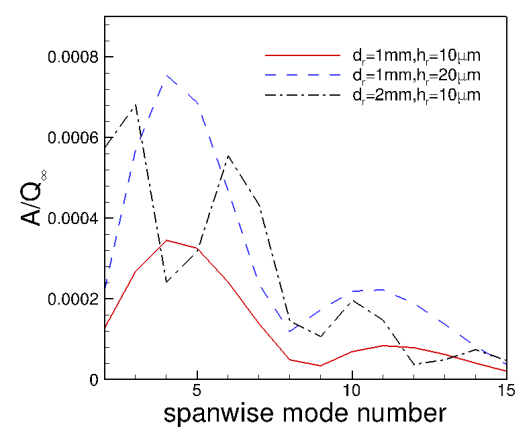

(a) $x / c=0.004$

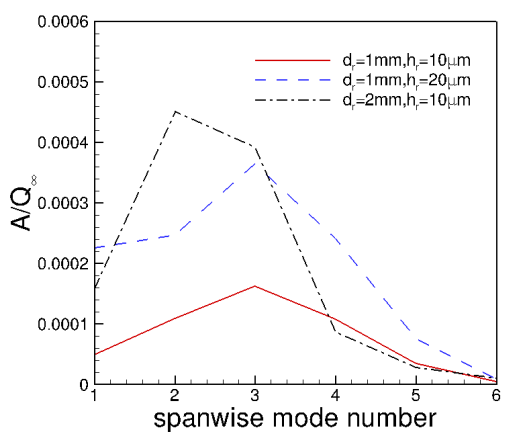

(b) $x / c=0.05$

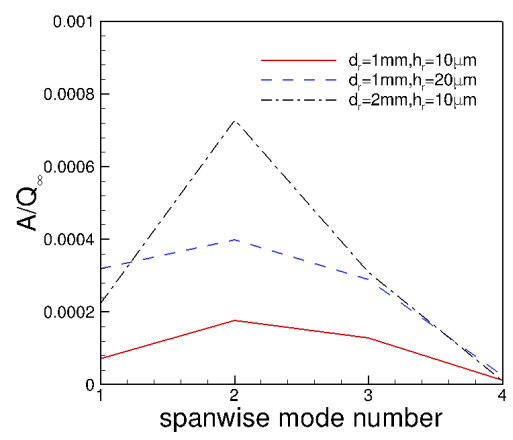

(c) $x / c=0.1$

Figure 8. Initial spanwise wavenumber spectra of the maximum chordwise velocity perturbation for all roughness elements: (a) $x / c=0.004$; (b) $x / c=0.05$; (c) $x / c=0.1$.

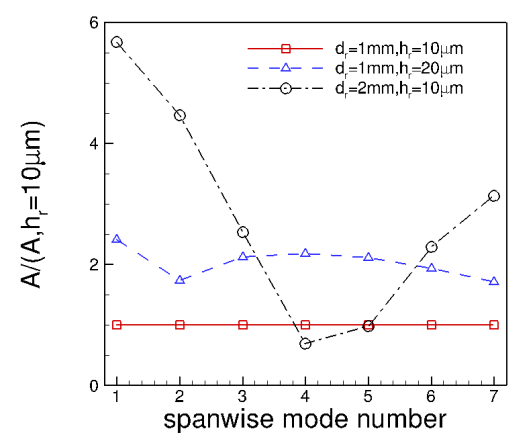

(a) $x / c=0.004$

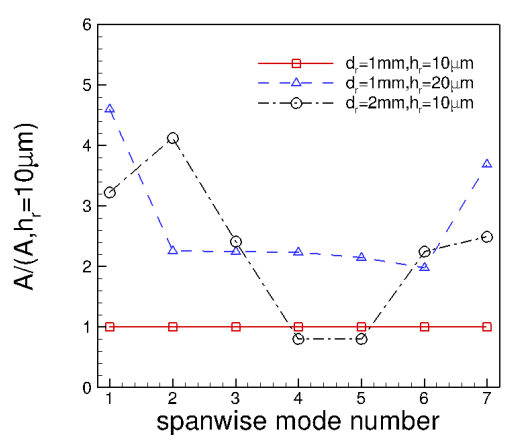

(b) $x / c=0.05$

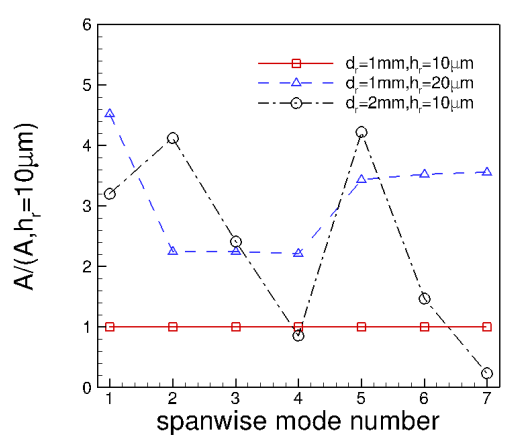

(c) $x / c=0.1$

Figure 9. Nondimensional initial spanwise wavenumber spectra of the maximum chordwise velocity perturbation for all roughness elements, with the amplitude of each mode normalized by the corresponding amplitude of the Case Cyl_D1_H1_L8p5 $\left(d_{r}=1 \mathrm{~mm}, h_{r}=10 \mu \mathbf{m}\right):(\mathbf{a}) x / c=0.004$; (b) $x / c=0.05 ;$ (c) $x / c=0.1$.



(a)

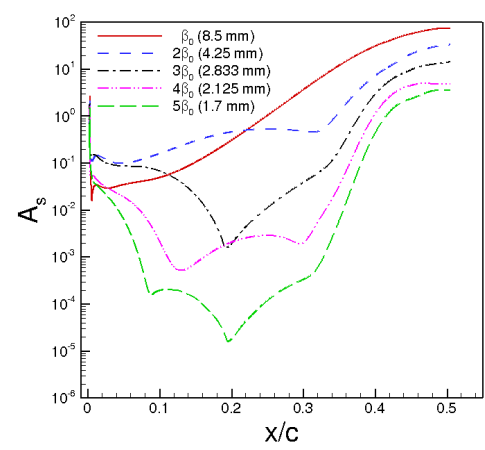

(b)

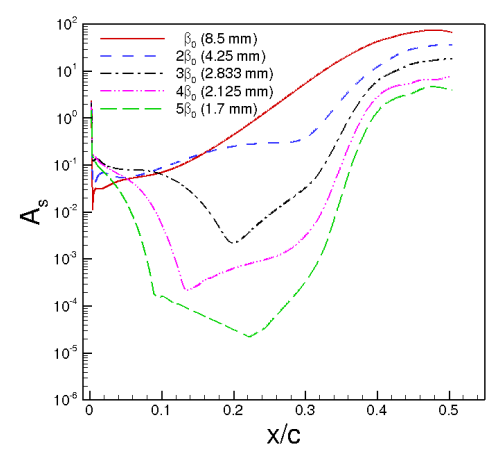

(c)

Figure 10. Disturbance amplitudes $A_{s}$ of the fundamental mode $\beta_{0}(8.5-\mathrm{mm}$ mode) and the first four superhamonics $\left(2 \beta_{0}, 3 \beta_{0}, 4 \beta_{0}, 5 \beta_{0}\right)$ as predicted by DNS for cylindrical roughness elements with a spanwise spacing of $L_{r}=8.5 \mathbf{m m}$. (a) $d_{r}=1 \mathrm{~mm}, h_{r}=10 \mu \mathbf{m}$; (b) $d_{r}=2 \mathrm{~mm}, h_{r}=10 \mu \mathbf{m}$; (c) $d_{r}=1 \mathrm{~mm}, h_{r}=20 \mu \mathbf{m}$. 


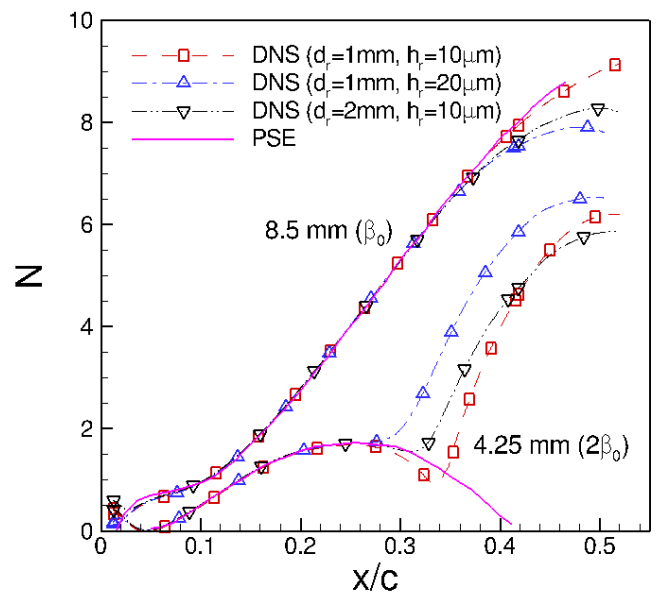

Figure 11. Logarithmic-amplification ratios $N$ of the fundamental mode $\beta_{0}$ and the first superharmonic $2 \beta_{0}$ as predicted by DNS and linear PSE for roughness cases with $L_{r}=8.5 \mathrm{~mm}$.

Here $h_{r}$ denotes the wall-normal displacement whose magnitude can be adjusted with the value of $\epsilon_{r} ; \beta_{0}$ and $L_{y}$ is the spanwise wavenumber and DNS domain size, respectively; $x_{s}$ and $x_{e}$ denote the starting and ending chordwise locations of the roughness strip, respectively; $L_{1}$ and $L_{2}$ are the lengths of the smoothing zone at the start and end of the roughness, respectively; $S$ is a smooth step function defined in Schrader et al..; ${ }^{25}$ and $\varphi_{n}$ is the modal phase chosen randomly. Because of the spanwise periodic boundary conditions, only disturbances with a wavenumber of $n \beta_{0}(n=1,2, \ldots)$ can be accounted for in the DNS. Figure 12 provides a schematic of the model parameters. The roughness model, referred to in this paper as "natural" surface roughness, is implemented as inhomogeneous boundary conditions by projecting the no-slip conditions on the surface of the roughness to the undisturbed wall using Taylor-series expansions as ${ }^{26}$

$$
\begin{aligned}
\left.u\right|_{w} & =-h_{r}\left(\left.\frac{\partial \bar{u}}{\partial n}\right|_{w}\right) \\
\left.v\right|_{w} & =-h_{r}\left(\left.\frac{\partial \bar{v}}{\partial n}\right|_{w}\right) \\
\left.w\right|_{w} & =-h_{r}\left(\left.\frac{\partial \bar{w}}{\partial n}\right|_{w}\right) \\
\left.T\right|_{w} & =\left.\bar{T}\right|_{w}-h_{r}\left(\left.\frac{\partial \bar{T}}{\partial n}\right|_{w}\right) .
\end{aligned}
$$

Table 3 lists the values of the model parameters for the DNS of receptivity to natural surface roughness. The roughness parameters are determined based on the lessons learned from the study of receptivity to cylindrical roughness elements as discussed in Section B. In particular, the mode number $M N$ is chosen to be 5 as the DNS of receptivity to cylindrical roughness elements show that only the 8.5 -mm mode $\left(\beta_{0}\right)$ and the first four superhamonics $\left(2 \beta_{0}, 3 \beta_{0}, 4 \beta_{0}, 5 \beta_{0}\right)$ grow significantly (Figure 10).

Figures 13a and 13b show the chordwise evolution of disturbance amplitudes $A_{s}$ for the DNS cases with natural surface roughness NatH10 and NatH1, respectively, while Figure 14 shows the logarithmicamplification ratios $N$. Receptivity of the laminar swept-wing boundary layer is sensitive to the distribution of roughness, with the initial amplitude of the crossflow disturbances excited by natural roughness significant larger than that induced by cylindrical roughness of the same height. The 8.5 -mm mode $\left(\beta_{0}\right)$ and the first four superhamonics excited by natural surface roughness show a chordwise growth similar to those forced by cylindrical roughness before they become saturated due to nonlinear effects; the $8.5-\mathrm{mm}$ mode becomes dominant over all the smaller wavelength modes after $x / c=0.2$ and remain unstable over longer streamwise disturbances, while all the smaller wavelength modes grow first (i.e., at small distance from the wing leading edge) but decay farther downstream. Among all the superhamonics, the 4.25 -mm mode $\left(2 \beta_{0}\right)$ and the 2.833 - 
mm mode $\left(3 \beta_{0}\right)$ experience strong initial growth and their initial amplitudes are maintained over a relatively large streamwise distance up to $x / c \approx 0.2$.

Table 3. Summary of roughness parameters for DNS study of receptivity to distributed natural surface roughness.

\begin{tabular}{cccccccc}
\hline Case & $\epsilon_{r}(\mu \mathrm{m})$ & $L_{y}(\mathrm{~mm})$ & $M N$ & $x_{s} / c$ & $x_{e} / c$ & $L_{1} / c$ & $L_{2} / c$ \\
NatH10 & 10 & 8.5 & 5 & $-0.854 \%$ & $0.131 \%$ & $1.31 \times 10^{-3}$ & $2.61 \times 10^{-4}$ \\
NatH1 & 1 & 8.5 & 5 & $-0.854 \%$ & $0.131 \%$ & $1.31 \times 10^{-3}$ & $2.61 \times 10^{-4}$ \\
\hline
\end{tabular}

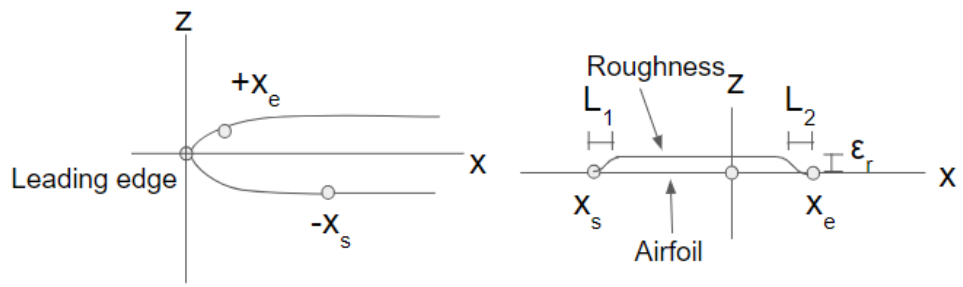

Figure 12. Schematic of the model parameters for natural roughness used in the DNS.

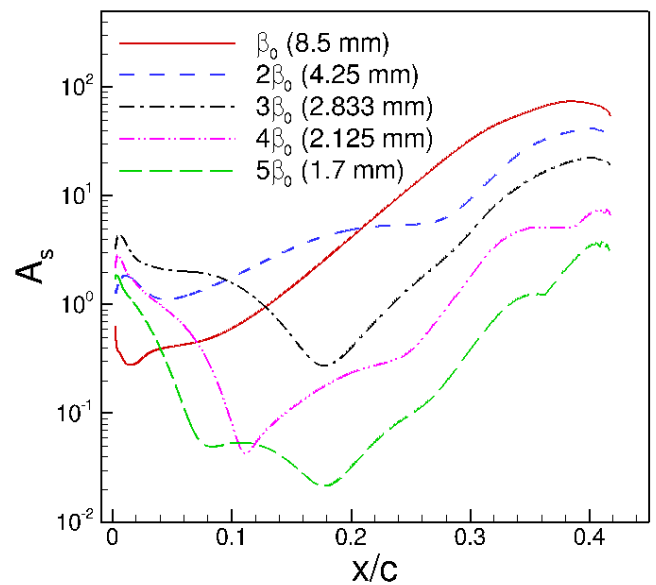

(a)

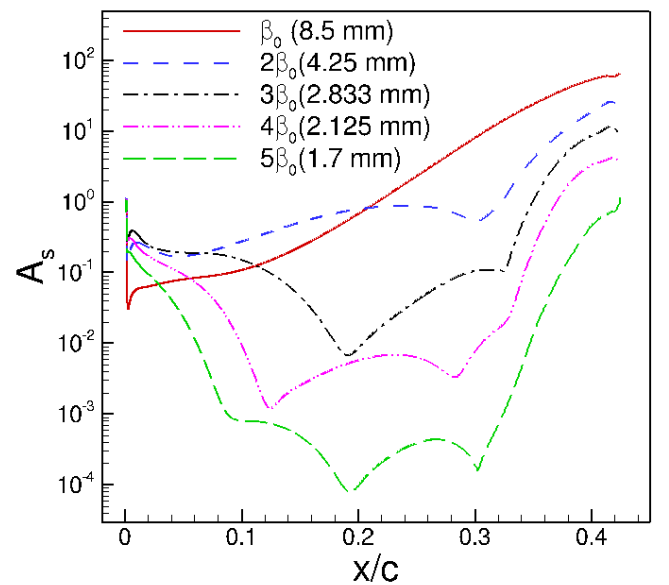

(b)

Figure 13. Disturbance amplitudes $A_{s}$ as predicted by DNS for distributed natural surface roughness (a) Case NatH10 (b) Case NatH1.

\section{Summary}

Direct numerical simulations (DNS) are performed to examine the receptivity to roughness in a spatially developing subsonic swept-wing boundary layer over an infinite-swept natural-laminar-flow wing configuration (GII-B, TAMU-0706) at high Reynolds numbers relevant to transport aircraft. The surface roughness is implemented in the DNS either as discrete meshed cylinders combined with cyclic boundary conditions, which mimics a spanwise periodic row of roughness elements, or as an appropriate simplified roughness model combined with inhomogeneous boundary conditions at the undisturbed wall, which mimics naturally occurring distributed roughness. Roughness parameters are varied in the DNS to explore the relation between the size and distribution of roughness and the induced crossflow disturbances in the boundary layer. The main observations and conclusions summarized as follows: 


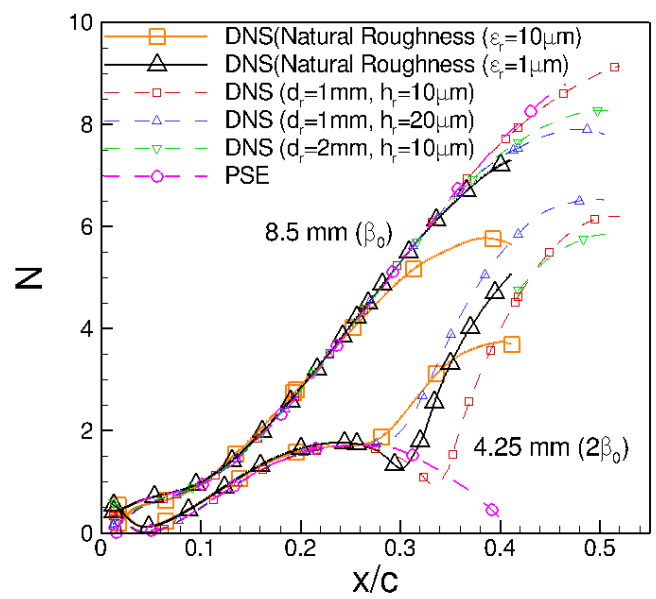

Figure 14. Logarithmic-amplification ratios $N$ as predicted by DNS for distributed natural surface roughness.

(i) Both cylindrical and natural roughness elements can excite a wide spectrum of spanwise modes.

(ii) The initial spanwise spectral content is highly dependent upon the shape of roughness elements.

(iii) The initial growth of the crossflow structures is a nonlinear function of the element height.

(iv) The linear growth of the excited unstable crossflow disturbances predicted by DNS shows good agreement with linear PSE.

The receptivity study lays the foundation for investigating the stabilization of the naturally most unstable steady crossflow mode by using spanwise periodic DREs. Currently DNS that implement both natural roughness and control cylinders are being conducted; the DNS simulate a typical experiment scenario in which multiple steady crossflow modes including the most unstable mode (i.e., the "target" mode) emerge because of the presence of naturally distributed surface roughness in the leading edge region and spanwise periodic control cylinders of subcritical wavelength are used to force small-wavelength disturbances (i.e., the control mode) for damping the target mode. The DNS results will be used to show the effectiveness of DREs in attenuating the naturally most unstable steady crossflow mode at high Reynolds numbers relevant to transport aircraft.

\section{Acknowledgments}

This work was originally sponsored under the NASA Environmentally Responsible Aviation Project.

\section{References}

\footnotetext{
${ }^{1}$ Carpenter, A. L., Saric, W. S., Reed, H. L., and Saric, W. S., "Laminar Flow Control on a Swept Wing with Distributed Roughness," AIAA Paper 2008-7335, 2008.

${ }^{2}$ Saric, W. S., Carpenter, A. L., and Reed, H. L., "Passive Control of Transition in Three-Dimensional Boundary Layers, with Emphasis on Discrete Roughness Element," Phil. Trans. R. Soc. A, Vol. 369, No. 1940, 2011, pp. $1352-1364$.

${ }^{3}$ Saric, W. S., Carrillo, R. B., and Reibert, M. S., "Nonlinear Stability and Transition in 3-D Boundary Layers," Meccanica, Vol. 33, 1998, pp. 469-487.

${ }^{4}$ Saric, W. S., Carrillo, R. B., and Reibert, M. S., "Leading-edge Roughness as a Transition Control Mechanism," AIAA Paper 1998-0781, 1998.

${ }^{5}$ Malik, M. R., Li, F., Choudhari, M. M., and Chang, C. L., "Secondary Instability of Crossflow Vortices and Swept-Wing Boundary-Layer Transition," Journal of Fluid Mechanics, Vol. 399, 1999, pp. 85-115.

${ }^{6}$ Haynes, T. S. and Reed, H. L., "Simulation of Swept-Wing Vortices using Nonlinear Parabolized Stability Equations," Journal of Fluid Mechanics, Vol. 405, 2000, pp. 325-349.
} 
${ }^{7}$ Wassermann, P. and Kloker, M., "Mechanisms and Passive Control of Crossflow-Vortex-Induced Transition in a ThreeDimensional Boundary Layer," Journal of Fluid Mechanics, Vol. 456, 2002, pp. 49-84.

${ }^{8}$ Rizzetta, D. P., Visbal, M. R., Reed, H. L., and Saric, W. S., "Direct Numerical Simulation of Discrete Roughness on a Swept-Wing Leading Edge," AIA A Journal, Vol. 48, No. 11, 2010, pp. 2660-2673.

${ }^{9} \mathrm{Li}$, F., Choudhari, M. M., Chang, C.-L., Streett, C., and Carpenter, M., "Computational Study of Laminar Flow Control on a Subsonic Swept Wing Using Discrete Roughness Elements," AIAA Journal, Vol. 49, No. 3, 2011, pp. $520-529$.

${ }^{10}$ Hosseini, S. M., Tempelmann, D., Hanifi, A., and Henningson, D. S., "Stabilization of a Swept-Wing Boundary Layer by Distributed Roughness Elements," Journal of Fluid Mechanics, Vol. 718, 2013, pp. R1-R11.

${ }^{11}$ Belisle, M. J., Roberts, M. W., Tufts, M. W., Tucker, A. A., Williams, T., Saric, W. S., and Reed, H. L., "Design of the Subsonic Aircraft Roughness Glove Experiment (SARGE)," AIAA Paper 2011-3524, 2011.

${ }^{12}$ Malik, M., Liao, W., Li, F., and Choudhari, M., "Discrete-Roughness-Element-Enhanced Swept-Wing Natural Laminar Flow at High Reynolds Numbers," AIAA Journal, Vol. 53, No. 8, 2015, pp. 2321-2334.

${ }^{13} \mathrm{Li}$, F., Choudhari, M. M., Carpenter, M., Malik, M., Chang, C.-L., and Streett, C., "Control of Crossflow Transition at High Reynolds Numbers Using Discrete Roughness Elements," AIAA Journal, Vol. 54, No. 1, 2016, pp. 39-52.

${ }^{14}$ Piot, E., Content, C., and Casalis, G., "Receptivity of Crossflow Instabilities to a Periodic Roughness Array on a Swept Cylinder: Investigation of the Roughness Size Influence," AIAA Paper 2008-0502, 2008.

${ }^{15}$ Tempelmann, D., Schrader, L. U., Hanifi, A., Brandt, L., and Henningson, D. S., "Swept Wing Boundary-Layer Receptivity to Localized Surface Roughness," Journal of Fluid Mechanics, Vol. 711, 2012, pp. 516-544.

${ }^{16}$ Collis, S. S. and Lele, S. K., "Receptivity to Surface Roughness Near a Swept Leading Edge," Journal of Fluid Mechanics, Vol. 380, 1999, pp. 141-168.

${ }^{17}$ Thomas, C., Mughal, S., and Ashworth, R., "On Predicting Receptivity to Surface Roughness in a Compressible Infinite Swept Wing Boundary Layer," Physics of Fluids, Vol. 29, No. 3, 2017, pp. 034102.

${ }^{18}$ Reibert, M. S., Nonlinear Stability, Saturation, and Transition in Crossflow-Dominated Boundary Layers, Ph.D. thesis, Arizona State University, 1996.

${ }^{19}$ Tufts, M. W., Reed, H. L., and Saric, W. S., "Design of an Infinite-Swept-Wing Glove for In-Flight Discrete-RoughnessElement Experiment," Journal of Aircraft, Vol. 51, No. 5, 2014, pp. 1618-1631.

${ }^{20} \mathrm{Li}, \mathrm{F}$. and Choudhari, M. M., "Spatially Developing Secondary Instabilities and Attachment Line Instability in Supersonic Boundary Layers," AIAA Paper 2008-590, 2008.

${ }^{21}$ Jiang, L., Choudhari, M. M., Chang, C. L., and Liu, C., "Direct Numerical Simulations of Crossflow Disturbances in Supersonic Boundary Layers," AIAA Paper 2004-589, 2004.

${ }^{22}$ Duan, L., Choudhari, M. M., and Li, F., "Direct Numerical Simulation of Transition in a Swept Boundary Layer," AIAA Paper 2013-2617, 2013.

${ }^{23}$ Jiang, G. S. and Shu, C. W., "Efficient Implementation of Weighted ENO Schemes," Journal of Computational Physics, Vol. 126, No. 1, 1996, pp. 202-228.

${ }^{24}$ Williamson, J., "Low-Storage Runge-Kutta Schemes," Journal of Computational Physics, Vol. 35, No. 1, 1980 , pp. $48-56$.

${ }^{25}$ Schrader, L. U., Brandt, L., and Henningson, D. S., "Receptivity Mechanisms in Three-Dimensional Boundary Layer Flows," Journal of Fluid Mechanics, Vol. 618, 2009, pp. 209-241.

${ }^{26}$ Choudhari, M. and Streett, C. L., "A Finite Reynolds-number Approach for the Prediction of Boundary-Layer Receptivity in Localized Regions," Phys. Fluids A, Vol. 4, No. 11, 1992, pp. 2495-2514. 